\title{
Soft X-ray emission lines from a relativistic accretion disk in MCG -6-30-15 and Mrk 766*
}

\author{
G. Branduardi-Raymont ${ }^{1}$, M. Sako ${ }^{2}$, S. M. Kahn ${ }^{2}$, A. C. Brinkman ${ }^{3}$, J. S. Kaastra ${ }^{3}$, and M. J. Page ${ }^{1}$ \\ 1 Mullard Space Science Laboratory, University College London, Holmbury St. Mary, Dorking, Surrey, \\ RH5 6NT, UK \\ 2 Department of Physics and Columbia Astrophysics Laboratory, Columbia University, 550 West 120th Street, \\ New York, NY 10027, USA \\ 3 Space Research Organisation of The Netherlands, Sorbonnelaan 2, 3584 CA Utrecht, The Netherlands
}

Received 2 October 2000 / Accepted 6 November 2000

\begin{abstract}
XMM-Newton Reflection Grating Spectrometer (RGS) spectra of the Narrow Line Seyfert 1 galaxies MCG -6-30-15 and Mrk 766 are physically and spectroscopically inconsistent with standard models comprising a power-law continuum absorbed by either cold or ionized matter. We propose that the remarkably similar features detected in both objects in the 5-35 $\AA$ band are H-like oxygen, nitrogen, and carbon emission lines, gravitationally redshifted and broadened by relativistic effects in the vicinity of a Kerr black hole. We discuss the implications of our interpretation, and demonstrate that the derived parameters can be physically self-consistent.
\end{abstract}

Key words. black hole physics - accretion, accretion disks - line: formation - galaxies: individual: MCG -6-30-15 - galaxies: individual: Mrk 766 - X-rays: galaxies

\section{Introduction}

The precise shape of the low energy spectra of active galaxies has traditionally been very difficult to establish. The combined effects of interstellar absorption, moderate spectral resolution of available detectors, and intrinsic complexity in the sources have so far prevented us from determining even whether the underlying spectrum is mainly due to continuum emission, or includes discrete emission and absorption components. The model generally adopted to match the observations is that of a continuum spectrum absorbed by partially ionized material (Halpern 1984; Reynolds 1997, and references therein); the origin and location of this warm absorber at the core of active galaxies, however, is still very much a matter of debate (e.g., Otani et al. 1996).

The enhancement in energy resolution and sensitivity afforded by the XMM-Newton Reflection Grating Spectrometer (RGS; den Herder et al. 2001) provides us with the potential to unravel the true origin of the soft $\mathrm{X}$-ray emission in AGN for the first time. RGS observations of MCG -6-30-15 and Mrk 766, which are reported here, have forced us to examine alternatives to the warm absorber model, and to propose a new and radically

Send offprint requests to: G. Branduardi-Raymont

* Based on observations obtained with XMM-Newton, an ESA science mission with instruments and contributions directly funded by ESA Member States and the USA (NASA). different interpretation of the soft X-ray spectra of active galaxies.

\section{MCG -6-30-15 and Mrk 766}

MCG -6-30-15 and Mrk 766 (NGC 4253) are classified as Narrow Line Seyfert 1 (NLS1) galaxies on the basis of the widths of their Balmer lines $\left(<2000 \mathrm{~km} \mathrm{~s}^{-1}\right)$, although they are not of the extreme kind. Both show strong and rapid variability in their X-ray fluxes, as well as variability in the slope of their power-law continua. MCG -630-15 is not known to possess a "soft excess" (which is one of the dominant characteristics of this class of objects), while Mrk 766 displays a soft excess that varies less than the continuum at higher energies. Evidence for Compton reflection has been found only in MCG -630-15. Broad features in the $<1 \mathrm{keV}$ spectra of both sources have been attributed to absorption in an ionized interstellar medium at some distance from the central massive black hole. The profile of the broad fluorescent $\mathrm{Fe} \mathrm{K} \alpha$ line observed at $6-7 \mathrm{keV}$ can be explained as due to the effects of relativistic motions and gravitational redshift in a disk surrounding the central black hole (Tanaka et al. 1995). MCG -6-30-15 and Mrk 766 are bright $\left(L_{2-10 \mathrm{keV}} \sim\right.$ few $\left.10^{43} \mathrm{erg} \mathrm{s}^{-1}\right)$ and nearby $\operatorname{AGN}(z=0.00775 \pm 0.00005$ and $0.01293 \pm 0.00005$ for MCG -6-30-15 and Mrk 766, respectively; redshifts based on optical emission line measurements (Fisher et al. 1995; 


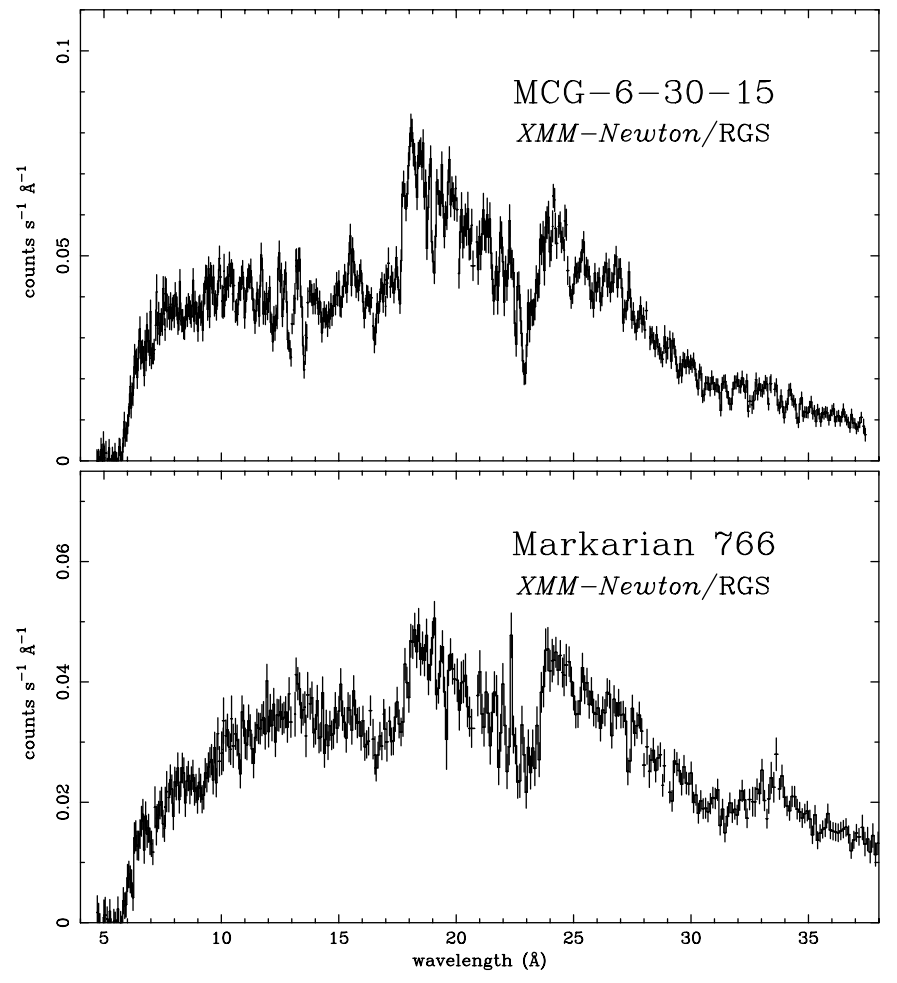

Fig. 1. The raw RGS first order spectra of MCG -6-30-15 (top) and Mrk 766 (bottom), plotted in the observer's frame

Smith et al. 1987), with relatively low Galactic absorption along the lines of sight $\left(4.110^{20} \mathrm{~cm}^{-2}\right.$ and $1.810^{20} \mathrm{~cm}^{-2}$, respectively).

\section{RGS observations and data analysis}

MCG -6-30-15 was observed with XMM-Newton in July 2000 for a total of $120 \mathrm{ks}$; Mrk 766 in May 2000 for $55 \mathrm{ks}$. The RGS data were processed with the XMM-Newton Science Analysis Software. Source and background events were extracted by making spatial and order selections on the event files, and were calibrated by applying the most up-to-date calibration parameters. The current wavelength scale is accurate to $\sim 8 \mathrm{~m} \AA$. The instrumental oxygen edge feature near $\lambda \sim 23 \AA$ mentioned in den Herder et al. (2001) was calibrated using observations of a pure continuum source, PKS 2155-304.

The raw extracted spectra are shown in Fig. 1. They are remarkably similar, in their overall shape and in the details, being dominated by prominent "saw-tooth" features, which peak at around 15, 18, 24 and $33 \AA$. A single power-law fit with neutral absorption is clearly an unacceptable representation of the observed spectra. In particular, the neutral oxygen edge at $23 \AA$ implies a higher column density than can be accommodated by the fit to the continuum. In addition, the spectra do not show neutral absorption edges from the other elements at their expected positions.

\subsection{Spectral fits with warm absorber models}

We attempted to fit the spectra with a warm absorber model which includes the appropriate absorption edges and absorption lines associated with all ions of abundant elements ( $, \mathrm{N}, \mathrm{O}, \mathrm{Ne}, \mathrm{Mg}$, and $\mathrm{Fe}$ ). The absorption line equivalent widths depend on the velocity profile, and we assume a turbulent velocity, which is a free parameter for each charge state. The absorbing column density of each charge state, as well as the neutral Galactic column density, are also left free to vary. The fits (shown in Fig. 2; best fit power-law slopes $\Gamma=2.14$ and 2.53 for MCG -6-30-15 and Mrk 766 respectively) are unacceptable for both objects, for a number of reasons. Firstly, the observed, putative "O VIII and O VII edges" are redshifted with respect to their expected positions (14.23 $\AA$ and $16.78 \AA$ for O viII and O viI, respectively), and by very large amonts $(\sim 1 \AA)$, corresponding to infall velocities on the order of $\sim 16000 \mathrm{~km} \mathrm{~s}^{-1}$. However, the absence of the associated absorption lines at the redshift implied by the edges for these two charge states places an upper limit in the line equivalent widths of $E W \sim 20 \mathrm{~m} \AA$. For the derived column densities of $N_{\text {OVIII }} \sim 410^{18} \mathrm{~cm}^{-2}$ and $N_{\text {OVII }} \sim 310^{18} \mathrm{~cm}^{-2}$ determined from the edges, these absorption lines are in the saturated region of the curve of growth. Therefore, the upper limit to the equivalent width implies a sensitive upper limit to the velocity width of the infalling material, which is $\leq 60 \mathrm{~km} \mathrm{~s}^{-1}$ for both objects. This is very difficult to reconcile with the apparent redshifts. The radial inflow, in this case, would have to be at one particular velocity. Re-emission following absorption is an unlikely explanation for the absence of the absorption lines. If the surrounding material is falling towards the nucleus, most of the material will be re-emitting radiation at shorter wavelengths than that of the absorbed resonance line, and we would expect to observe an inverted P Cygni profile, which is definitely not seen in the data.

The fits described above still require a significant neutral absorbing component in excess of the Galactic column density to these sources. In the case of Mrk 766, the neutral oxygen edge is again too high with respect to what is required to fit the data at longer wavelengths. An excess of flux is also present between 18 and $19 \AA$ in MCG -6-30-15.

\subsection{Disk-line emission interpretation}

The physical and spectroscopic implausiblities described above force us to examine alternative models to reproduce the observed RGS spectra. Remarkably, we have been able to obtain acceptable fits to both the MCG -6-30-15 and Mrk 766 data with a completely different model consisting of an absorbed power-law and emission lines, which are gravitationally redshifted and broadened by relativistic effects in a medium which is encircling a massive, rotating black hole. In this interpretation, the saw-toothed features in Fig. 1 are attributed to (in ascending wavelength order) H-like Ly $\alpha$ lines of O vIII, N VII, and C VI. 


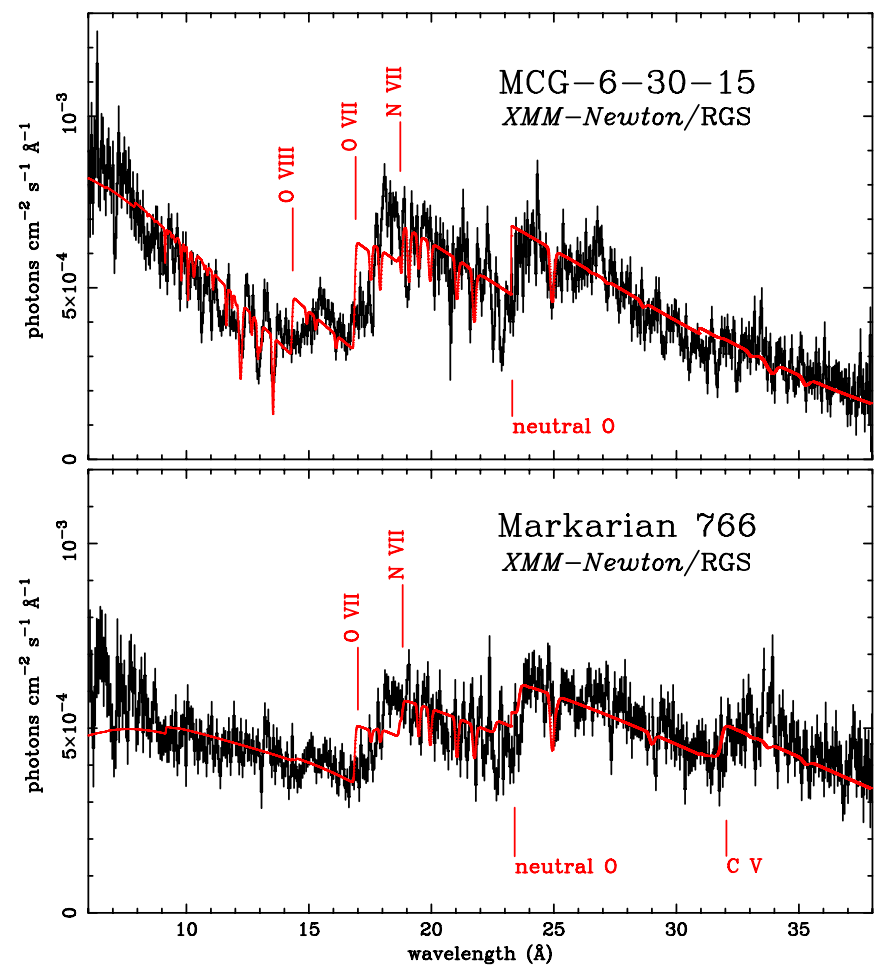

Fig. 2. "Fluxed" spectra of the two sources (corrected for effective area) with the best-fit warm-absorber model, plotted in the observer's frame

Our model includes a power-law continuum, with cold absorption fixed at the Galactic value, and three emission lines represented by profiles originating near a maximally rotating Kerr black hole (Laor 1991). The line wavelengths are fixed at their expected values in the observer's frame for the redshifts of the sources. The continuum power-law slope (photon index $\Gamma$ ) is fitted, as are the disk inclination angle $i$, the emissivity index $q$ (i.e., the slope of the radial emissivity profile in the disk), and the inner and outer limits $R_{\text {in }}$ and $R_{\text {out }}$ of the disk emission region. These parameters are tied for all the lines in the fit.

The best fit parameters are listed in Table 1; data and best fit models are shown in Fig. 3 for both MCG -6-30-15 and Mrk 766. The errors quoted correspond to $90 \%$ confidence ranges for one interesting parameter. The derived emissivity index of $q \sim 4$ indicates that most of the line emission originates from the inner part of the disk where gravitational effects are the strongest. The outer emission radius is, therefore, not well-constrained. For the same reason, disk emission line profiles produced in a Schwarzschild metric (Fabian et al. 1989) do not provide an acceptable fit to the data, since the last stable orbit, in this case, is substantially larger than that in the Kerr metric.

In our initial investigations of this model, we considered that the bump at $\sim 16 \AA$ in the spectrum of MCG -6$30-15$ could be $\mathrm{O}$ VIII Ly $\beta$ emission. However, we have concluded that this is unlikely, and do not model this feature as a relativistic $\mathrm{O}$ VIII $\mathrm{Ly} \beta$ emission line for reasons related to the physical self-consistency of our model. These reasons are explained in the following section, and

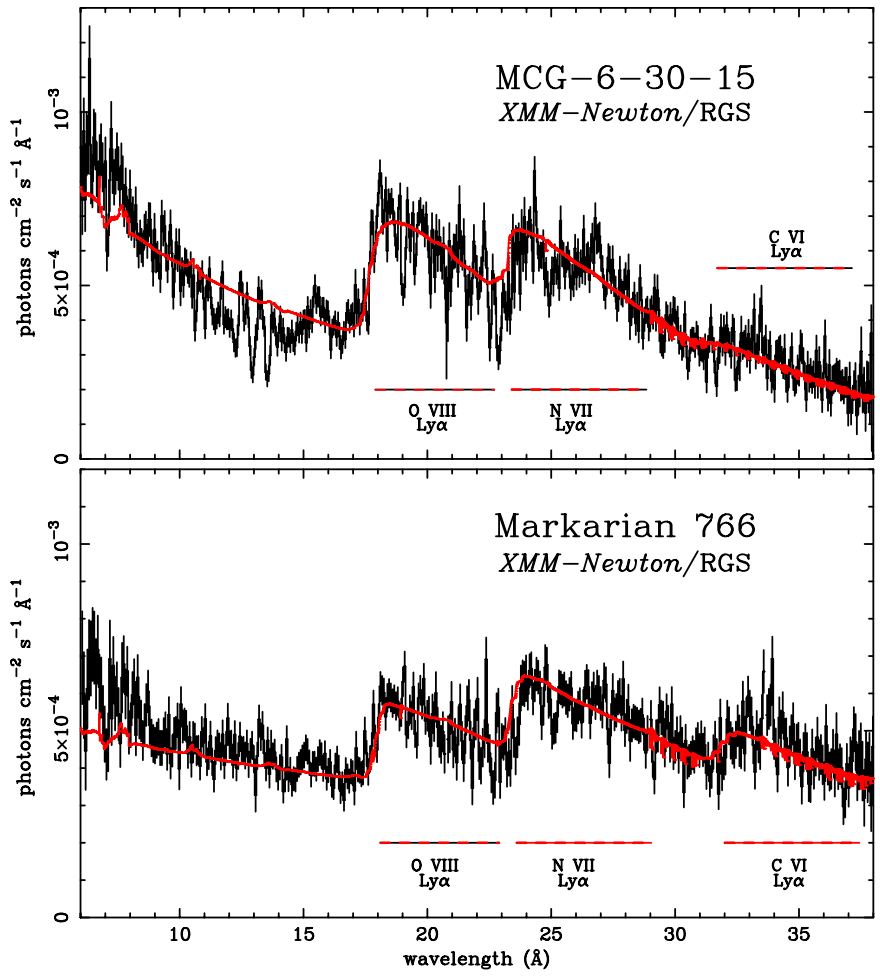

Fig. 3. Same as in Fig. 2 with the relativistically broadened line model. The parameters are listed in Table 1

Table 1. RGS best fit parameters for isolated lines model

\begin{tabular}{lll}
\hline \hline Parameter & MCG $-6-30-15$ & Mrk 766 \\
\hline \hline$\Gamma$ & $1.33 \pm 0.012$ & $1.68 \pm 0.03$ \\
Inclination $i$ & $40.3^{\circ} \pm 0.3^{\circ}$ & $35.6^{\circ} \pm 0.9^{\circ}$ \\
Emissivity index $q$ & $3.78 \pm 0.05$ & $3.56 \pm 0.09$ \\
$R_{\text {in }}$ & $1.24_{-0.0}^{+0.7}$ & $1.24_{-0.0}^{+0.8}$ \\
$R_{\text {out }}$ & $110_{-60}^{+90}$ & $60_{-15}^{+60}$ \\
C vi Ly $\alpha^{\mathrm{a}}$ & $(6.8 \pm 1.3) 10^{-4}$ & $(1.2 \pm 0.1) 10^{-3}$ \\
N vil Ly $^{\mathrm{a}}$ & $(2.4 \pm 0.1) 10^{-3}$ & $(1.9 \pm 0.1) 10^{-3}$ \\
O viII Ly $^{\mathrm{a}}$ & $(4.6 \pm 0.1) 10^{-3}$ & $(1.9 \pm 0.1) 10^{-3}$ \\
\hline
\end{tabular}

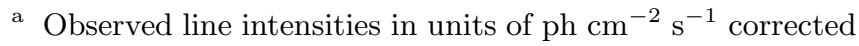
for Galactic absorption.

an alternative explanation for the $\sim 16 \AA$ feature will be discussed.

It is worth stressing again that both galaxies exhibit essentially an identical spectral structure, with multiple broadened lines of H-like oxygen, nitrogen, and carbon. The line energies are consistent with the galaxies systemic velocities, and all lines are consistent with having the same broad profiles. The fit residuals are also much less obvious and systematic than for the warm absorber model. In addition, the disk line parameters are consistent with those derived for the Fe $\mathrm{K} \alpha$ line $\left(i=34_{-6}^{\mathrm{o}}{ }_{-6}\right.$ and $36^{\circ}{ }_{-7}^{+8}, q=2.8 \pm 0.5$ and $3.0_{-0.4}^{+0.8}$ for MCG $-6-30-15$ and Mrk 766 respectively, Nandra et al. 1997). No additional 
column density to the Galactic value is required by the fits. All of these factors, which are consistent with each other to a degree that makes chance coincidences unlikely, imply that the relativistic line model is the most probable explanation for the present observations.

The models in Fig. 3 deliberately do not include narrow absorption features, in order to emphasize the quality of the line emission fit. We have re-fitted the spectra including, in addition to the three emission lines, absorption components from carbon, nitrogen, oxygen, neon, and iron. Lines from Ne IX-X, Fe XIX-XXI, O VII-VIII, N VII, and $\mathrm{C}$ VI are detected in MCG -6-30-15, while only oxygen, nitrogen, and carbon lines appear in Mrk 766. In contrast to the pure warm absorber fit, the absorption lines profiles are well-reproduced by the model with much lower ion column densities $\left(N_{i} \leq 10^{17} \mathrm{~cm}^{-2}\right)$ and a higher velocity width $\left(F W H M v_{\text {turb }} \sim 2000 \mathrm{~km} \mathrm{~s}^{-1}\right)$. The observed line positions in MCG -6-30-15 are slightly blueshifted from their rest wavelengths indicating outflow velocities of $v \sim 400 \mathrm{~km} \mathrm{~s}^{-1}$, while those in Mrk 766 are consistent with no net velocity shift. With such low column densities, no edges are expected to be detectable, as observed. The fits including these narrow lines are shown in Fig. 4.

\section{Physical consistency of the relativistic disk emission model?}

The lack of Fe L and He-like K emission lines in the RGS spectra suggests that the observed emission lines are most likely due to radiative recombination onto fully stripped ions (oxygen is fully ionized for $k T \geq 100 \mathrm{eV}$; Kallman \& Krolik 1995). In addition to the Ly $\alpha$ lines, one might expect to also see higher Lyman series as well as narrow radiative recombination continua ( $\mathrm{RRC})$. The spectra are definitely not consistent with the emission pattern expected when all of these additional features are included at the theoretical fluxes relative to that of the Ly $\alpha$ lines. However, as we describe below, the emitting plasma is most likely optically thick to both the photoelectric continuum and to the Lyman series themselves. We, therefore, adopt the measured Ly $\alpha$ line fluxes as discussed in the previous section to estimate the emission measures of each of the ions.

For a maximally rotating black hole with $i \sim 40^{\circ}$ and $q \sim 4$, most of the emitted flux is beamed away from the observer, and a correction factor is applied to the isotropic luminosity. Adopting the method of Cunningham (1975), we find $F_{\text {obs }} \sim 0.3 L_{\mathrm{em}} / 4 \pi D^{2}$, where $F_{\text {obs }}$ is the observed flux, $L_{\mathrm{em}}$ is the emitted luminosity in the rest frame of the emitting material, and $D$ is the distance to the source. The derived ion emission measures $\left(E M_{i+1}=n_{\mathrm{e}} n_{i+1} V\right)$ for Hlike carbon, nitrogen, and oxygen are listed in Table 2, where we have used recombination line powers as described in Liedahl \& Paerels (1996).

For a plasma in which the material is nearly fully stripped, the ratios of the ion $E M \mathrm{~s}$ provide the abundance ratios directly. The observed ratios are
Table 2. Ion emission measures derived from self-consistent fits

\begin{tabular}{lll}
\hline Ion & MCG $-6-30-15$ & Mrk 766 \\
\hline CVI Ly $\alpha^{\mathrm{a}}$ & $(0.54 \pm 0.13) T_{100 \mathrm{eV}}^{0.65}$ & $(3.6 \pm 0.4) T_{100 \mathrm{eV}}^{0.65}$ \\
NVII Ly $\alpha^{\mathrm{a}}$ & $(2.5 \pm 0.1) T_{100 \mathrm{eV}}^{0.65}$ & $(6.6 \pm 0.6) T_{100 \mathrm{eV}}^{0.65}$ \\
OVIII Ly $^{\mathrm{a}}$ & $(3.3 \pm 0.1) T_{100 \mathrm{eV}}^{0.82}$ & $(5.5 \pm 0.5) T_{100 \mathrm{eV}}^{0.82}$ \\
\hline
\end{tabular}

a Ion EMs in units of $10^{63} \mathrm{~cm}^{-3}$.

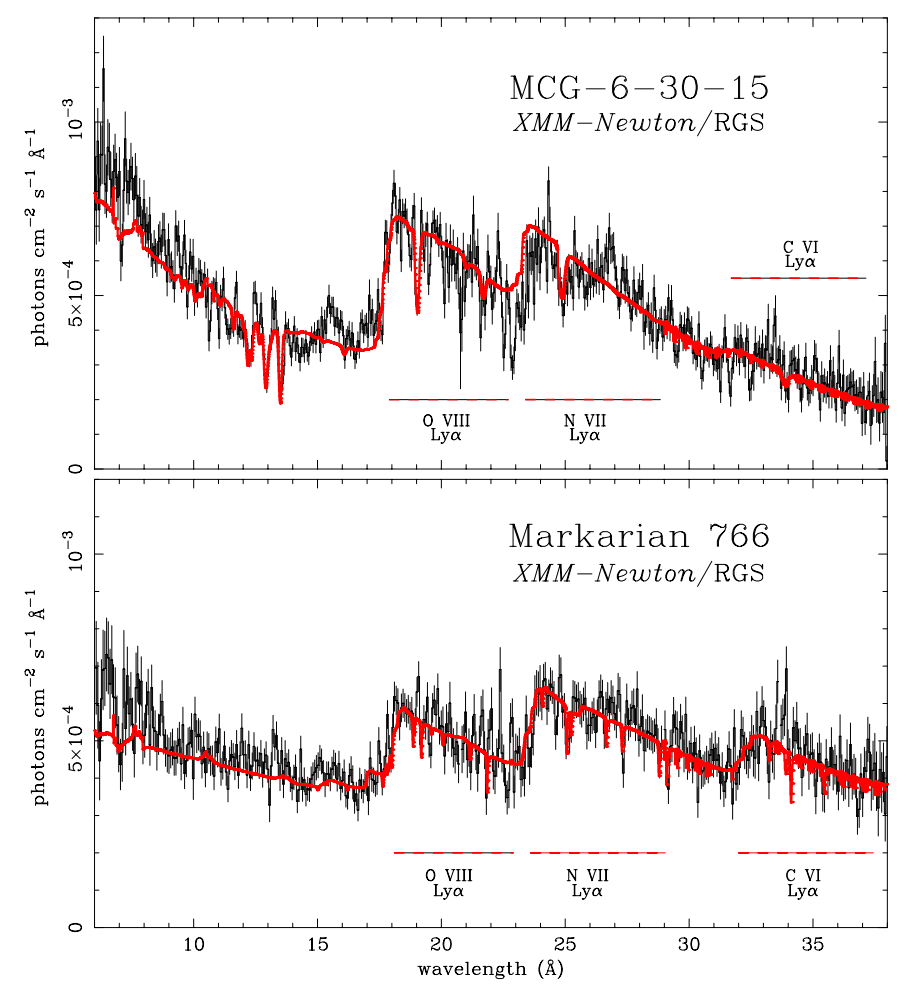

Fig. 4. Same as in Fig. 3 with the narrow absorption components

$A_{\mathrm{C}} / A_{\mathrm{O}}=0.16 \pm 0.04$ and $A_{\mathrm{N}} / A_{\mathrm{O}}=0.76 \pm 0.05$ for MCG $-6-30-15$, and $A_{\mathrm{C}} / A_{\mathrm{O}}=0.65 \pm 0.2$ and $A_{\mathrm{N}} / A_{\mathrm{O}}=$ $1.2 \pm 0.3$ for Mrk 766 . These ratios are rather different (particularly for Mrk 766) from the solar values of $A_{\mathrm{C}} / A_{\mathrm{O}}=0.43$ and $A_{\mathrm{N}} / A_{\mathrm{O}}=0.13$ (Anders \& Grevesse 1989). However, the strength of the nitrogen absorption lines in both MCG -6-30-15 and Mrk 766 suggests that nitrogen is overabundant in the extended absorbing medium as well. Such anomalies have also been inferred from UV observations of quasars (e.g., Hamann \& Ferland 1992; Artymowicz et al. 1993, and references therein).

Using the derived parameters for MCG -6-30-15, we calculate the abundance-corrected total emission measure $\left(E M=n_{\mathrm{e}}^{2} V\right)$ for O VIII to be,

$E M=3.810^{66} T_{100 \mathrm{eV}}^{0.82} A_{\mathrm{O} \odot}^{-1}$,

where $A_{\mathrm{O} \odot}$ is the oxygen abundance relative to the solar value.

Assuming a disk-like geometry, we estimate the total emission measure to be $E M \sim \pi n_{\mathrm{e}}^{2} R^{2} H$, where $R$ is 
the characteristic radius and $H$ is the scale height of the emitting material. In the inner regions of a relativistic accretion disk where the pressure is dominated by radiation, the ratio of the scale height to the disk radius is on the order of $f=H / R \sim 10^{-3}$ (Kato et al. 1998). Assuming that the characteristic emission radius is $R \sim 10 R_{\mathrm{g}}$, where $R_{\mathrm{g}}=G M / c^{2}=1.510^{12} M_{7} \mathrm{~cm}$ and $M_{7}$ is the mass of the black hole in multiples of $10^{7} M_{\odot}$, we can estimate the average electron density to be,

$n_{\mathrm{e}}=6.010^{14} T_{100 \mathrm{eV}}^{0.41} A_{\mathrm{O} \odot}^{-1 / 2} f_{-3}^{-1 / 2} M_{7}^{-3 / 2} \mathrm{~cm}^{-3}$,

where $f_{-3}$ is the ratio $H / R$ in multiples of $10^{-3}$. The implied electron scattering optical depth in the vertical direction of the disk is,

$\tau_{\mathrm{e}} \sim n_{\mathrm{e}} H \sigma_{\mathrm{T}}=6.0 T_{100 \mathrm{eV}}^{0.41} A_{\mathrm{O} \odot}^{-1 / 2} f_{-3}^{1 / 2} M_{7}^{-1 / 2}$.

These simple estimates may be uncertain by as much as a factor of a few.

The moderate to high optical depth may present a potential problem. For $\tau_{\mathrm{e}} \gtrsim 5$, broadening of the spectral lines due to electron scattering becomes comparable to the broadening from gravitational and relativistic effects. If $\tau_{\mathrm{e}} \lesssim 1$, however, electron scattering produces a negligible effect on the observed line profiles, and this situation is possible if, for example, the emission region is much smaller than the scale height of the accretion disk (i.e., $f_{-3} \ll 1$ ).

On the other hand, a medium in which $\tau_{\mathrm{e}} \sim 1$ is required to explain the absence of the RRC and the higher Lyman series lines. With trace abundances of the H-like species, the medium can be optically thick to its own RRC. In this case, recombination to the ground state is suppressed and most of the expected RRC flux is eventually radiated in the $\mathrm{Ly} \alpha$ line. It only takes an optical depth of order a few at the photoelectric edge in order to achieve this. In $\mathrm{O}$ viII, the threshold optical depth in the $\mathrm{K}$ edge is $\tau_{\text {OVIII }} \sim 130 \tau_{\mathrm{e}} A_{\mathrm{O} \odot} f_{\text {OVIII }}$, where $f_{\text {OVIII }}$ is the fractional ion abundance of O viII. Therefore, even a small trace abundance of $\mathrm{O}$ VIII can almost completely suppress the RRC. Moreover, since the medium is optically thick to photoelectric absorption, it is very optically thick to line absorption as well. The higher series Lyman lines (Ly $\beta$ and higher) are also destroyed by a mechanism similar to the one responsible for suppressing the RRC, since the upper levels can decay through channels other than to the ground level. The Ly $\alpha$ line, on the other hand, can decay only to the ground level and, therefore, is not destroyed.

Since the Ne X and Ne IX emission line wavelengths are close to but shorter than that of the O VIII edge, most of these line photons are probably also absorbed by O VIII, which subsequently are pumped into the O VIII Ly $\alpha$ line. The O viII Ly $\alpha$ line wavelength is longer than that of the N VII edge, and is not affected by this opacity effect. However, the N VII Ly $\alpha$ line is just on the short wavelength side of the $\mathrm{C}$ VI edge, and might be somewhat affected.

A medium with $\tau_{\mathrm{e}} \sim 1$ is also an efficient reflector, which suggests that a large fraction of the illuminating continuum radiation is also reflected into our line of sight. With trace elements of $\mathrm{H}$-like oxygen, for example, the reflected spectrum should contain an absorption edge feature that is also distorted by strong relativistic effects. Therefore, the residual feature near $\lambda \sim 15-16 \AA$ in MCG -6-30-15, may be identified as an O viII edge, analogous to the iron $\mathrm{K}$ edge absorption feature produced in reflection from a cold medium. The precise characterization of these opacity effects, however, requires a detailed radiative transfer calculation with self-consistent photoionization models, which is beyond the scope of this Letter.

To check for consistency in the parameters derived above, we compute the upper limit for the average ionization parameter of the plasma to be,

$\xi=\frac{L}{n_{\mathrm{e}} R^{2}}=710^{3} L_{43} T_{100 \mathrm{eV}}^{-0.41} A_{\mathrm{O} \odot}^{1 / 2} f_{-3}^{1 / 2} M_{7}^{-1 / 2}$,

which is consistent with the observed level of ionization in that $\mathrm{C}, \mathrm{N}$, and $\mathrm{O}$ should be nearly fully stripped. The temperature at this level of ionization is $k T \sim 200 \mathrm{eV}$.

The same calculations have been applied to Mrk 766, with the following results:

$E M=6.310^{66} T_{100 \mathrm{eV}}^{0.82} A_{\mathrm{O} \odot}^{-1}$,

$n_{\mathrm{e}}=7.810^{14} T_{100 \mathrm{eV}}^{0.41} A_{\mathrm{O} \odot}^{-1 / 2} f_{-3}^{-1 / 2} M_{7}^{-3 / 2} \mathrm{~cm}^{-3}$,

$\tau_{\mathrm{e}}=7.8 T_{100 \mathrm{eV}}^{0.41} A_{\mathrm{O} \odot}^{-1 / 2} f_{-3}^{1 / 2} M_{7}^{-1 / 2}$.

Note that the emission measures derived from the observed O VIII Ly $\alpha$ line fluxes are overestimated due to the opacity effect, which, as described above, enhances the line fluxes compared to the case of an optically thin, purely recombining plasma. Since all of the line power radiated by elements with atomic number $Z>8$, as well as the higher series lines in oxygen, are eventually pumped into $\mathrm{O}$ VIII Ly $\alpha$, the true intrinsic emission measures may be lower by as much as a factor of $\sim 3$ compared to those estimated in Eqs. (1) and (5).

The observed RGS spectra require a flattening of the underlying continuum radiation in both MCG -6-30-15 and Mrk 766 below $\sim 2.5 \mathrm{keV}$. A preliminary spectral analysis of the EPIC PN data of MCG -6-30-15 shows that a power-law slope of $\Gamma \sim 1.3$ can reproduce the 1-2 keV spectrum, with substantial excess soft emission below $\sim 1 \mathrm{keV}$. A simple extension of the PN 3-10 keV continuum (power-law slope $\Gamma=1.97_{-0.03}^{+0.02}$ ) down to lower energies also requires excess emission below $\sim 1 \mathrm{keV}$. Most of the observed soft X-ray flux, however, is in the form of $\mathrm{C}, \mathrm{N}$, and $\mathrm{O}$ emission lines. If the hard X-ray continuum radiation is produced through inverse Compton scattering primarily of these line photons, the apparent break in the photon index may be a natural consequence (Sunyaev \& Titarchuk 1980).

Clearly the results we present here and their interpretation in terms of line emission from a relativistic disk call for a complete re-assessment of the processes leading to the production of high energy radiation in the cores of 
active galaxies. Such a study will have to account for the flattening of the hard X-ray continuum towards low energies as well as the detailed line-formation processes in the inner regions of the accretion disk.

\section{Conclusions}

We have shown that a simple warm-absorber interpretation of the RGS spectra of MCG -6-30-15 and Mrk 766 is untenable on spectroscopic grounds. Broad line emission from a relativistic disk surrounding a maximally rotating Kerr black hole seems to explain the data remarkably well. The physical self-consistency of this scenario remains to be established, however, the preliminary analysis presented in Sect. 4 is encouraging. Note that the conclusions we draw do not depend on any pre-conceived assumption about the shape of the ionizing continuum.

This result could not have been achieved without the combination of large effective area and high energy resolution afforded by the XMM-Newton RGS. The poorer resolution of CCD spectrometers cannot provide discriminatory power for the warm absorber versus line emission debate raised by the RGS results presented in this paper. A more robust test will be finding the same problems and applying the same solution to other AGN sources.

Acknowledgements. The authors would like to thank Duane Liedahl for kindly providing the atomic calculations. The Mullard Space Science Laboratory acknowledges financial support from the UK Particle Physics and Astronomy Research Council. The Columbia University team is supported by NASA. The Laboratory for Space Research Utrecht is supported financially by the Netherlands Organization for Scientific Research (NWO).

\section{References}

Anders, E., \& Grevesse, N. 1989, Geochim. Cosmochim. Acta, 53,197

Artymowicz, P., Lin, D. N. C., \& Wampler, E. J. 1993, ApJ, 409, 592

Cunningham, C. T. 1975, ApJ, 202, 788

den Herder, J. W., Brinkman, A. C., Kahn, S. M., et al. 2001, A\&A, 365, L7

Fabian, A. C., Rees, M. J., Stella, L., \& White, N. E. 1989, MNRAS, 238, 729

Fisher, K. B., Huchra, J. P., Strauss, M. A., et al. 1995, ApJS, 100, 69

Halpern, J. P. 1984, ApJ, 281, 90

Hamann, F., \& Ferland, G. 1992, ApJ, 391, L53

Kallman, T. R., \& Krolik, J. H. 1995, XSTAR - A Spectral Analysis Tool, HEASARC (NASA/GSFC, Greenbelt)

Kato, S., Fukue, J., \& Mineshige, S. 1998, Black-hole Accretion Disks (Kyoto: Kyoto University Press)

Laor, A. 1991, ApJ, 376, 90

Liedahl, D. A., \& Paerels, F. 1996, ApJ, 468, L33

Nandra, K., George, I. M., Mushotzky, R. F., Turner, T. J., \& Yaqoob, T. 1997, ApJ, 477, 602

Otani, C., Kii, T., Reynolds, C. S., et al. 1996, PASJ, 48, 211

Reynolds, C. S. 1997, MNRAS, 286, 513

Smith, B. J., Kleinmann, S. G., Huchra, J. P., \& Low, F. J. 1987, ApJ, 318, 161

Sunyaev, R. A., \& Titarchuk, L. G. 1980, A\&A, 86, 121

Tanaka, Y., Nandra, K., Fabian, A. C., et al. 1995, Nat, 375, 659 